\title{
SEX DIFFERENCES IN MENTAL ROTATION STRATEGY ${ }^{1,2}$
}

\author{
SYLVIA RAABE, RAINER HÖGER, AND JUAN D. DELIUS \\ Experimental Psychology \\ University of Konstanz, Germany
}

\begin{abstract}
Summary.-When humans decide whether two visual stimuli are identical or mirror images of each other and one of the stimuli is rotated with respect to the other, the time discrimination takes usually increases as a rectilinear function of the orientation disparity. On the average, males perform this mental rotation at a faster angular speed than females. This experiment required the rotation of both mirror-image-different and non-mirror-different stimuli. The polygonal stimuli were presented in either spatially unfiltered, high-pass or low-pass filtered versions. All stimulus conditions produced mental rotation-type effects but with graded curvilinear trends. Women rotated faster than men under all conditions, an infrequent outcome in mental rotation studies. Overall, women yielded more convexly curvilinear response functions than men. For both sexes the curvilinearity was more pronounced under the non-mirrordifferent, low-pass stimulus condition than under the mirror different, high-pass stimulus condition. The results are considered as supporting the occurrence of two different mental rotation strategies and as suggesting that the women were predisposed to use efficiently an analytic feature rotation strategy, while the men were predisposed to employ efficiently a holistic pattern rotation strategy. It is argued that the overall design of this experiment promoted the application of an analytic strategy and thus conferred an advantage to the female participants.
\end{abstract}

When humans are required to judge whether two visual stimuli are identical or mirror images of each other and one of the stimuli is presented rotated with respect to the other, the response latencies - and oftentimes the error rates too-increase as a function of the orientation disparity between the stimuli. First described by Shepard and Metzler (1971) this effect has been often replicated and is one of the most reliable phenomena in cognitive psychology (Cooper, 1975; Hochberg \& Gelman, 1977; Shepard \& Cooper, 1982; Robertson \& Palmer, 1983; Corballis, 1988; Takano, 1989; Tarr \& Pinker, 1989). Most studies report essentially rectilinear response latency-orientation disparity functions but some authors have obtained markedly curvilinear functions (e.g., Jolicoeur, Regehr, Smith, \& Smith, 1985; Karadi, Csatho, Kallai, \& Labadi, 2003). As far as found, no cogent explanation has been offered for these curvilinearities. Participants frequently report that to

${ }^{1}$ Address correspondence to J. Delius, Algemeine Psychologie, Universität Konstanz, 78457 Konstanz, Germany or e-mail (juan.delius@uni-konstanz.de).

${ }^{2}$ The research was supported by the Deutsche Forschungsgemeinschaft, Bonn. We are grateful to Martina Siemann for essential collaboration and to Manuela Lehr for running part of the experiment. We also thank W. Nagl (Konstanz) for important statistical assistance and R. Hübner (Konstanz), and I. Rentschler (Munich) for technical advice. S. Raabe is presently with the Münchner Süd-Chemie A.G., Munich, and R. Höger is now at the Fachhochschule NordostNiedersachsen, Luneburg, Germany.

Konstanzer Online-Publikations-System (KOPS) URN: http://nbn-resolving.de/urn:nbn:de:bsz:352-206058 
decide whether the stimuli were equal or not, they resorted to rotating mentally one of the stimuli until both stimuli were identically orientated; hence, the term mental rotation, under which the effect is generally known. The mental rotation account has been supported by the findings that the motor cortex is activated during visual mental rotation tasks and that a concurrent manual rotation of a wheel or ball interferes with the performance of visual rotation tasks (Wohlschläger \& Wohlschläger, 1998; Richter, Somorjai, Summers, Jarmasz, Menon, Gati, Georgopoulos, Tegeler, Ugurbil, \& Kim, 2000; Tomasino, Borroni, Isaja, \& Rumiati, 2005).

It is commonly found that on the average men mentally rotate faster than do women (Linn \& Peterson, 1985; Voyer, Voyer, \& Bryden, 1995; Collins \& Kimura, 1997; Makany, Karadi, Kallai, \& Nadel, 2002; Karadi, et al., 2003; but see Tan, Okuyan, Albayrak, \& Akgun, 2003). This sex difference is mostly ascribed to the better visuospatial test scores generally exhibited by men in comparison with women. The disparity is said to have its ontogenetic origin in hormonal differences which in turn are rooted in genetic dissimilarities (Nyborg, 1983; Grimshaw, Sitarenios, \& Finnegan, 1995; Hausmann, Slabbekoorn, Van Goozen, Cohen-Kettenis, \& Güntürkün, 2000; Davison \& Susman, 2001). The genetic dissimilarities are again thought to go back to sex-differential selection pressures that acted during the phylogeny of hominoids (Mcburney, Gaulin, Devineni, \& Adams, 1997; Dabbs, Chang, Strong, \& Milun, 1998). It is nevertheless probable that cultural-educational differences also play a role in the development of the spatial ability advantage of men over women (Flaherty, 2005; Terlecki \& Newcombe, 2005).

However, it has been also suggested that the better spatial performance of men might only arise because commonly used spatial ability tests, including the mental rotation task, predominantly tap a holistic spatial ability whereas women may be prone to rely on an analytic spatial ability not specifically assessed by common spatial aptitude tests (Kylonnen, Lohman, \& Woltz, 1984; Hosenfeld, Strauss, \& Köller, 1997; see also Karadi, et al., 2003). In an earlier mental rotation study some results were obtained which might advance this approach. Förster, Gebhardt, Lindlar, Siemann, and Delius (1996) reported, contrary to a commonly held opinion (Zimmer \& Fröhlich, 1991; Pierret \& Perronet, 1994) but in agreement with less well known evidence (Folk \& Luce, 1987; Tarr \& Pinker, 1989), that non-mirror image, structurally arbitrarily different stimuli could also lead to mental rotationlike results. Some women in Förster, et al.'s experiment reported that they had solved this rotation-task variant by mentally rotating a local feature rather than the global stimulus pattern (unpublished data). Other authors have similarly suggested that mental rotation performances might occasionally be based on an analytic appraisal of detailed features rather than on a rotation 
of whole patterns (Yuile \& Steiger, 1982; Robertson \& Palmer, 1983; Jolicoeur, et al., 1985; Paquet, 1991; Kunde \& Hoffmann, 2000). Förster, et al. also noted that the rotation task using non-mirror-different stimuli tended to generate strongly convex curvilinear, rather than predominantly rectilinear, latency-disparity dependencies. This finding suggested that at high disparities of interstimulus orientation the non-mirror-stimuli could be mentally rotated faster than at low interstimulus-orientation disparities. The same authors also show that a rotation effect peaking at $90^{\circ}$ interstimulus disparity rather than at $180^{\circ}$ interstimulus orientation disparity could be obtained with extremely minimal visual stimuli, that is, using a comparison line that differed by an only small fraction in length from the sample reference line.

Förster, et al. (1996) proposed that the convex curvilinearity of the response latency-orientation disparity functions they had obtained with irregular, complex polygons might have come about through the concurrent application of an analytic rotation of component line features and of a holistic rotation of the total stimulus patterns. They also considered that the stronger curvilinearity they had obtained with non-mirror-different stimuli might reflect that these stimuli were more likely than mirror-different stimuli to elicit an analytic rather than a holistic same-different detection strategy (Schultz, 1991; Eme \& Marquer, 1999). This would be so because the former patterns could in principle be discriminated on the basis of only the size of a single figural element, whereas the latter could be discriminated only on the basis of the handedness relationship of at least two figural elements. The presumed faster detailistic, or less precisely expressed, "analytic processing might be increasingly applied to greater disparities in orientation for which the holistic processing would be comparatively slow. This circumstance would favor the emergence of a convex curvilinear, accelerating latency-disparity function with non-mirror-different stimuli. To this line of reasoning was added the assumption that the perception of global pattern shapes is mainly based on low spatial frequency information, whereas the perception of local features is assumed to rely mainly on high spatial frequency information (Navon, 1977; Shulman, Sulivan, Gish, \& Sakoda, 1986). Low frequency stimuli devoid of definite line and angle elements can thus be expected to promote a holistic rotation strategy, whereas high frequency stimuli which highlight these details can be expected to encourage an analytic rotation strategy.

An experimental condition which combined a non-mirror-different stimulus and high-pass filtered stimuli was hypothesized strongly to promote the emergence of curvilinear rotation functions presumed indicative of the incidence of an analytic style of rotation. In contrast with this, it was also hypothesized that a condition which combined a mirror-image-different stimulus and low-pass filtered stimuli would favor the occurrence of rectilinear 
rotation functions suggestive of a holistic mode of rotation. In conjunction with the earlier cited evidence that women are probably more adept at using an analytic spatial strategy than are men, the inclusion of the first condition was in turn expected to augment the overall mental rotation speed of the women.

\section{METHOD}

Subjects

Twenty female and 20 male university students or employees, ages 18 to 32 years, were either completing a course requirement or were paid for their participation. They rested the chin and forehead in a support frame $60 \mathrm{~cm}$ away from a $40-\mathrm{cm}$ diagonal flat-face monitor. The display system had an effective resolution of $2040 \times 1420$ pixels and a refresh rate of $70 \mathrm{~Hz}$. The keyboard of the computer was placed in front of and below the monitor. Before an experimental session began, the experimenter keyed up a written instruction on the monitor. It told the participants that they were going to be presented a series of stimulus triplets and that out of the two right- and left-flanking patterns they were to always choose the pattern that was identical to the central pattern by pressing one of two corresponding choice keys. Instructions also included a passage about responding as correctly and as promptly as possible.

Stimuli

The stimulus triplets were assembled from irregular polygons. Two different polygons served as alternative central sample stimuli and as corresponding identical (same) flanking comparison stimuli (Fig. 1, middle panels). For one of these two polygons, a structurally slightly modified, non-mirror-different polygon served as the different second flanking comparison stimulus. This polygon had been previously identified by Förster, et al. (1996) as being relatively hard to discriminate from the corresponding sample polygon. As these authors explained, this is a precondition for obtaining a mental rotation effect with such non-mirror-different polygons. For the other sample polygon, the different comparison polygon was its right-left mirrorimage. The sample polygon of each triplet appeared centered within the monitor display, with the two comparison polygons flanking it, centered 8.7 $\mathrm{cm}$ to the left and to the right. The individual polygons were approximately inscribable with an imaginary $2.2 \mathrm{~cm}$ diameter circle. To ensure task diversity and diminish influence of a practice effect (see Leone, Taine, \& Droulez, 1993), the sample polygon appeared alternatively in either an as originally drawn version or in an upside-down version. The flanking comparison polygons in turn both appeared with a $0,30,60,90,120,150$, or $180^{\circ}$ clockwise orientation disparity in relation to the relevant sample polygon. Each of the so assembled stimulus triplets occurred in a same-comparison polygon 
right-flanking, different-comparison polygon left-flanking, and in a samecomparison polygon left-flanking, different-comparison polygon right-flanking version.

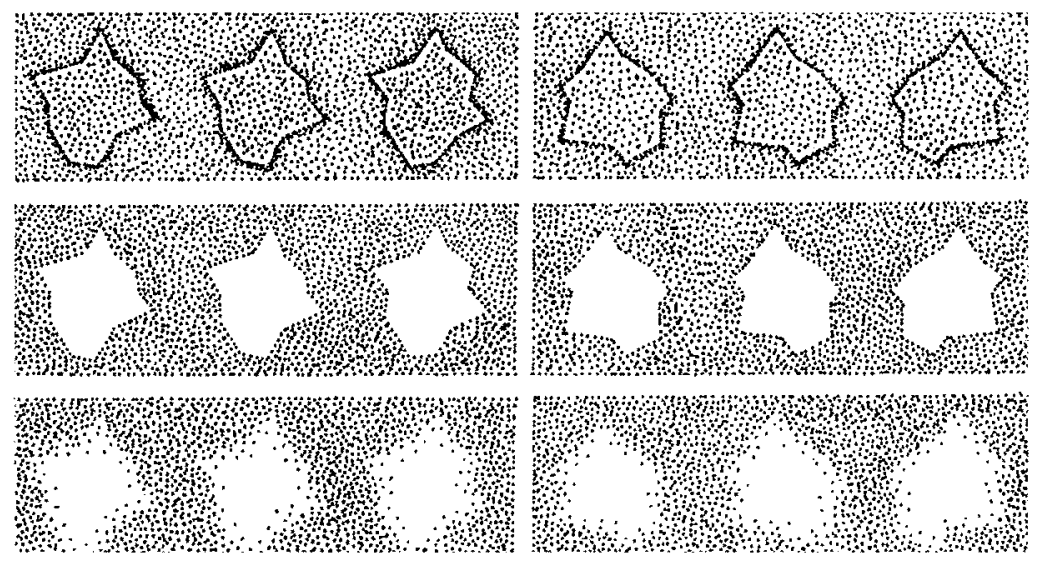

Fig. 1. Examples of stimulus triplets, all of the $0^{\circ}$ orientation disparity kind, and all of the same-polygon, left-flanking, different-polygon, right-flanking variety. Note that the actual experiment also involved triplets with $30,60,90,120,150$, and $180^{\circ}$ orientation disparities and triplets with an inverse tight-left flanking arrangement. Mirror-different comparison polygon triplets are shown in the left-side panels, and non-mirror-different comparison polygon triplets are shown in the right-side panels of the figure. High-pass filtered triplets are shown in the top row, unfiltered triplets in the middle row, and low-pass filtered triplets in the bottom row panels. Note that the black-white reproduction of the original gray-graded stimuli is only a rough one.

The triple- rather than the more usual twin-stimulus presentation was chosen to circumvent the occurrence of separate same-stimulus and different-stimulus detection trials that lead to complicating analysis and interpretation issues (cf. Kreuger, 1984; Farrel, 1988). In several experiments which employed only the usual unfiltered stimuli and mirror-image-different stimuli, the triple stimulus presentation technique has been shown to yield mental rotation results which are closely comparable (nearly rectilinear response-disparity functions, faster rotation by men) with those obtained with the more usually used twin stimulus-presentation technique (Hollard \& Delius, 1982; Förster, et al., 1996; and unpublished data of Raabe \& Delius). Moreover, Siemann (1997) using the twin stimulus technique closely replicated the results Förster, et al. had obtained with the triple technique. Hollard and Delius (1982) further observed that with the triple stimulus technique there was no difference in the mental rotation results whether human subjects were instructed to choose the same comparison stimulus or to choose the different comparison stimulus. 
Though as explained earlier the primary interest was in comparing the non-mirror-different, high-pass and mirror-different, low-pass condition, for counterbalancing purposes each, and all of the thus assembled stimulus triplets were presented in an unfiltered, a low-pass filtered, and a high-pass filtered variant (Fig. 1, top and lower row) creating a total of six stimulus conditions (see Fig. 3 below). The spatially filtered versions of the triplets were produced by convolving the digitized $(312 \times 312$ pixels) unfiltered polygons within background surrounds, with binomial filters (Höger, 1997). A Gaussian filter ( $\sigma=7$ pixels) was used to produce the low-pass stimulus variants. A difference of Gaussian filters ( $\sigma_{1}=0.5$ and $\sigma_{2}=0.01$ pixels) was used to produce the high-pass stimulus variants. As viewed by the subjects, the low-pass stimuli contained the less-than 3 cycles/degree spatial frequencies and appeared as blurred border figures while the high-pass stimuli contained the higher than 10 cycles/degree spatial frequencies and appeared as outline figures. All the stimuli were presented on a medium grey background. There were thus a total of 84 different stimulus triplets.

\section{Procedure}

Each session began with a warm-up presentation of five randomly selected triplets. During the session proper each of the 84 different triplets was presented eight times resulting in a total of 672 trials. The order of presentation of the triplets, including of course the same-right, different-left and same-left, different-right versions, was quasi-random (Gellermann, 1933). Subjects initiated the trials by pressing the down-pointing cursor key. A white $0.5-\times 0.5 \mathrm{~cm}$ fixation cross appeared centered on the monitor for 350 msec. The presentation of a stimulus triplet followed; the sample stimulus replaced the cross. The time which elapsed between the onset of the stimuli and the instant at which the subjects chose the left or right cursor keys was recorded as response latency. If the subject chose the sample-identical, correct comparison stimulus by pressing either the corresponding left- or the corresponding right-pointing cursor key, the stimulus triplet disappeared and $400 \mathrm{msec}$. later the word "richtig" (right) appeared centered on the monitor. If the subject chose the incorrect, different comparison stimulus (mirror or different), the stimulus triplet disappeared, and $400 \mathrm{msec}$. later the word "falsch" (wrong) appeared framed in red and accompanied by a 500-msec. buzzing tone. The message vanished when the subject initiated the next trial. After the session was over the subjects were told about the purpose of the experiment.

\section{Results}

Mean latencies and error rates were computed for each of the participants and each of the stimulus conditions. The mean latencies were log transformed and the error rates were arcsine transformed. They were then 
subjected to separate repeated-measurement analyses of variance with orientation disparity, subject's sex, stimulus type, and filtering factor as independent variables (Table 1; see also Fig. 3). Both latencies and errors were significantly affected by the orientation-disparity (latencies $F_{6,33}=55.38, p<001$; errors $F_{6,33}=7.73, p<.001$ ). Significant rectilinear and curvilinear trends were

TABLE 1

Analyses of Variance of Log Mean latencies and Arcsin Error Rates: Degrees of Freedom, Variance Quotients, and Statistical Significance

\begin{tabular}{|c|c|c|c|c|c|}
\hline \multirow[t]{2}{*}{ Effects } & \multirow[t]{2}{*}{$d f$} & \multicolumn{2}{|c|}{ Latencies } & \multicolumn{2}{|c|}{ Error Rates } \\
\hline & & $F$ & $p$ & $F$ & $p$ \\
\hline Orientation disparity & $6 / 33$ & 55.38 & .001 & 7.73 & .001 \\
\hline Participants' sex & $1 / 38$ & 1.28 & ns & .82 & ns \\
\hline Disparity $\cdot$ Sex & $6 / 33$ & 4.06 & .005 & 1.16 & ns \\
\hline Stimulus type & $1 / 38$ & .14 & ns & 6.45 & .05 \\
\hline Stimulus $\cdot$ Disparity & $6 / 33$ & 23.59 & .001 & 4.33 & .005 \\
\hline Stimulus $\cdot$ Sex & $1 / 38$ & .02 & ns & 1.63 & ns \\
\hline Stimulus $\cdot$ Disparity $\cdot$ Sex & $6 / 33$ & 1.61 & as & .90 & ns \\
\hline Filtering factor & $2 / 37$ & 2.33 & ns & 13.76 & .001 \\
\hline Filtering - Disparity & $12 / 27$ & 9.47 & .001 & 2.38 & .05 \\
\hline Filtering $\cdot$ Sex & $2 / 37$ & .19 & ns & .57 & ns \\
\hline Filtering $\cdot$ Stimulus & $2 / 37$ & .81 & ns & .38 & ns \\
\hline Filtering $\cdot$ Disparity $\cdot$ Sex & $12 / 27$ & .83 & ns & .28 & ns \\
\hline Filtering $\cdot$ Disparity $\cdot$ Stimulus & $12 / 27$ & 7.23 & .001 & .92 & ns \\
\hline Filtering $\cdot$ Sex $\cdot$ Stimulus & $2 / 37$ & .67 & ns & .76 & ns \\
\hline Filtering $\cdot$ Stimulus $\cdot$ Disparity $\cdot$ Sex & $12 / 27$ & .77 & ns & .94 & ns \\
\hline
\end{tabular}

evident for both the latencies $\left(F_{1,38}=251.82, p<.001 ; F_{1.38}=90.09, p<.001\right)$ and the errors $\left(F_{1.38}=43.21, p<.001 ; F_{1,38}=13.81, p<.001\right)$. There were no significant main effects for sex of subject affecting latencies or errors but there was a significant interaction for disparity $\times \operatorname{sex}$ for the latencies $\left(F_{6,33}=\right.$ $4.06, p<.005)$ but not for errors; the interaction affecting latencies involved significant rectilinear $\left(F_{1.38}=7.14, p<.05\right)$ and curvilinear $\left(F_{1.38}=6.05, p<.05\right)$ trends. The stimulus type factor significantly affected errors $\left(F_{1.38}=6.45, p<\right.$ .05) but not latencies. However, interactions for stimulus $\times$ disparity significantly affected both latencies and errors $\left(F_{6,33}=23.59, p<.001 ; F_{6,33}=4.33\right.$, $p<.005)$. The filtering factor affected errors $\left(F_{2.37}=13.76, p<.001\right)$ but not latencies; however, an interaction for filtering $\times$ disparity was significant for both latencies and errors $\left(F_{12,27}=9.47, p<.001 ; F_{12,27}=2.38, p<.05\right)$. Latencies $\left(F_{12,27}=7.23, p<.001\right)$, but not errors, were furthermore significantly affected by the interaction for filtering $\times$ disparity $\times$ stimulus. All other interactions were not significant ( $p s>.05$ ).

In agreement with significant curvilinear trends cited above, comparisons of the latency and error differences between the $0^{\circ}$ and $60^{\circ}$ orientation disparities and the differences between the $120^{\circ}$ and $180^{\circ}$ orientation dispar- 
ities across all 40 subjects regardless of sex showed that indeed the slopes of the latency-disparity and error-disparity function at smaller disparities were significantly steeper than at the larger disparities (latency: $t_{39}=9.61, p<.001$; errors $t_{39}=3.46, p<.001$; cf. Fig. 2 ). Contrary to various suggestions, the exclusion of all latencies pertaining to error choices, the exclusion of subjects with more than $8 \%$ choice errors, an exclusion of all latencies longer than $10 \mathrm{sec}$, and the use of medians rather than averages did not remove the convex curvature of the latency-disparity functions; it was a robust characteristic of the subjects' performance.

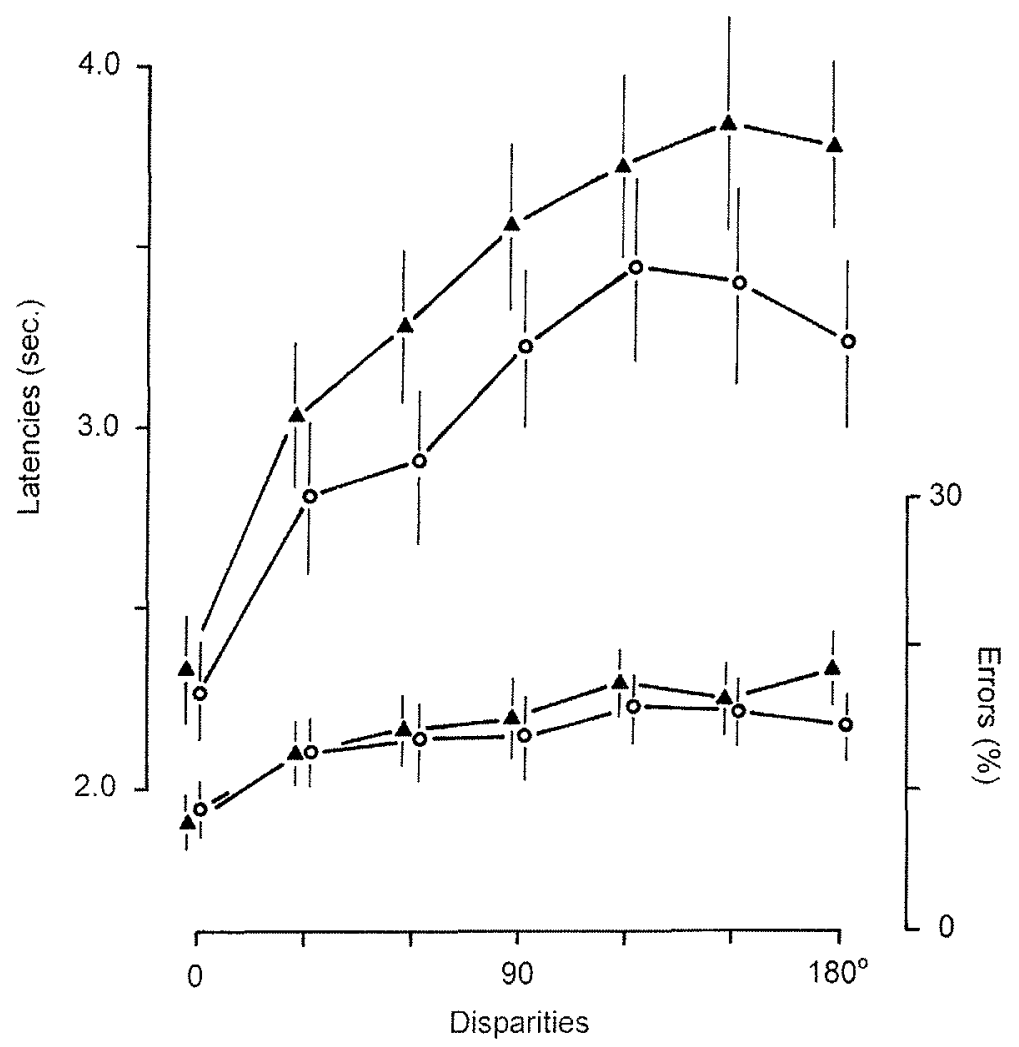

Fig. 2. Mean latencies (sec.) and error rates $(\%) \pm$ standard errors as a function of orientation disparity and sex (men: $\boldsymbol{\Lambda}$, women $O$ ) averaged across all six stimulus conditions.

Fig. 2 presents the most salient result of the experiment which was that averaged over all six stimulus conditions the women exhibited a flatter slope latency-disparity function, indicating a faster mental rotation, than the men (significant interaction for disparity $\times$ sex), while both sexes produced closely 
similar overall error rates (no significant interaction for disparity $\times$ sex). Furthermore, consistent with the significant curvilinear trend affecting the interaction of disparity $\times$ sex for latencies, the differences between the mean latencies corresponding to the $120^{\circ}$ and $180^{\circ}$ orientation disparities were significantly smaller for women than for men $\left(t_{37}=2.51, p<.01\right)$.

Fig. 3 shows the data of the experiment sorted according to the six stimulus conditions, with Fig. 4 singling out the latency-disparity and errordisparity functions obtained under the non-mirror-different, high-pass and mirror-different, low-pass conditions that earlier were said to be of special

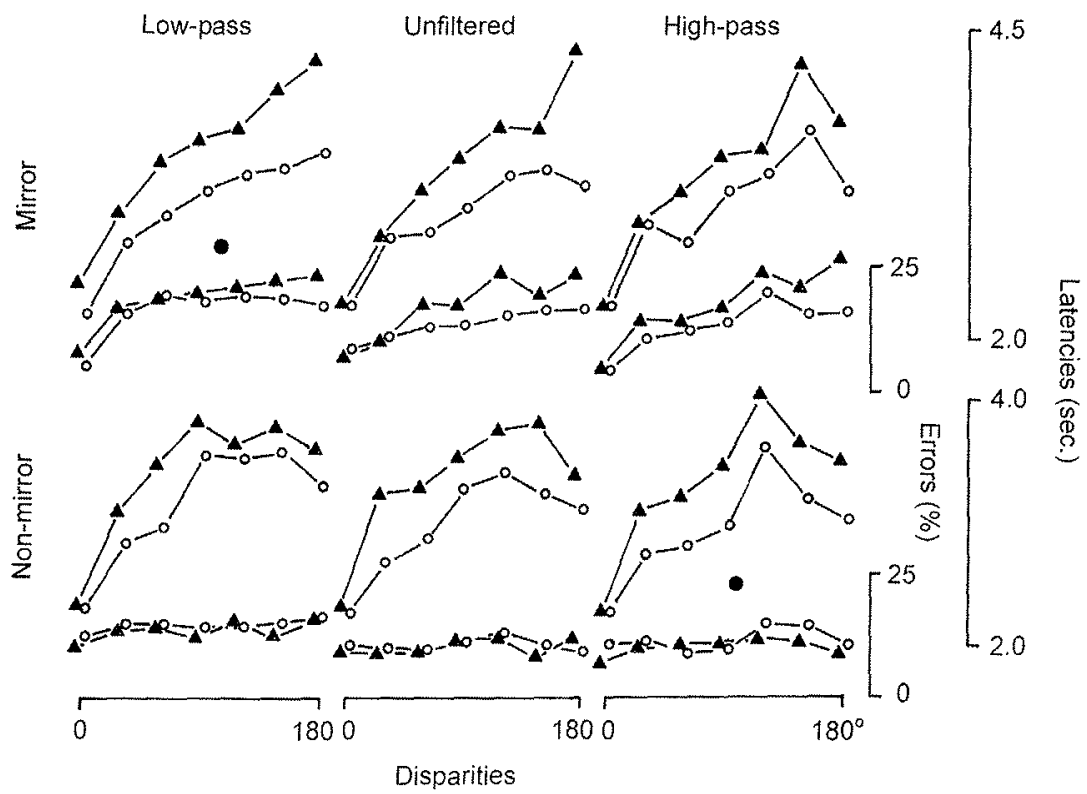

Fic. 3. Mean latencies (sec.) and crror rates (\%) plotted as functions of orientation disparities. Separately for men ( $\boldsymbol{\Delta})$ and women (O), for mirror-different (upper row panels) and non-mirror-different stimulus types (lower row panels) and for the three different stimulus-filtering conditions (left, middle, and right column panels). Panels $(\bullet)$ are shown enlarged in Fig. 4.

interest. Averaged over both conditions the mean latency differences between the $120^{\circ}$ and $180^{\circ}$ orientation disparities were significantly smaller for women than for men $\left(t_{37}=2.51, p<05\right)$. For both men and women the latency differences between the $120^{\circ}$ to $180^{\circ}$ orientation disparities were significantly smaller under the mirror-different, low-pass stimulus condition than under the non-mirror-different, high-pass stimulus condition (men: $t_{19}=4.39$, $p<001$; women: $t_{10}=3.10, p<.01$ ). However, there was no significant differ- 
ence between men and women concerning these two particular conditions as to the latency differences between the $120^{\circ}$ and $180^{\circ}$ orientation disparities. There were no significant differences in all these respects concerning error rates (cf. Fig. 4).

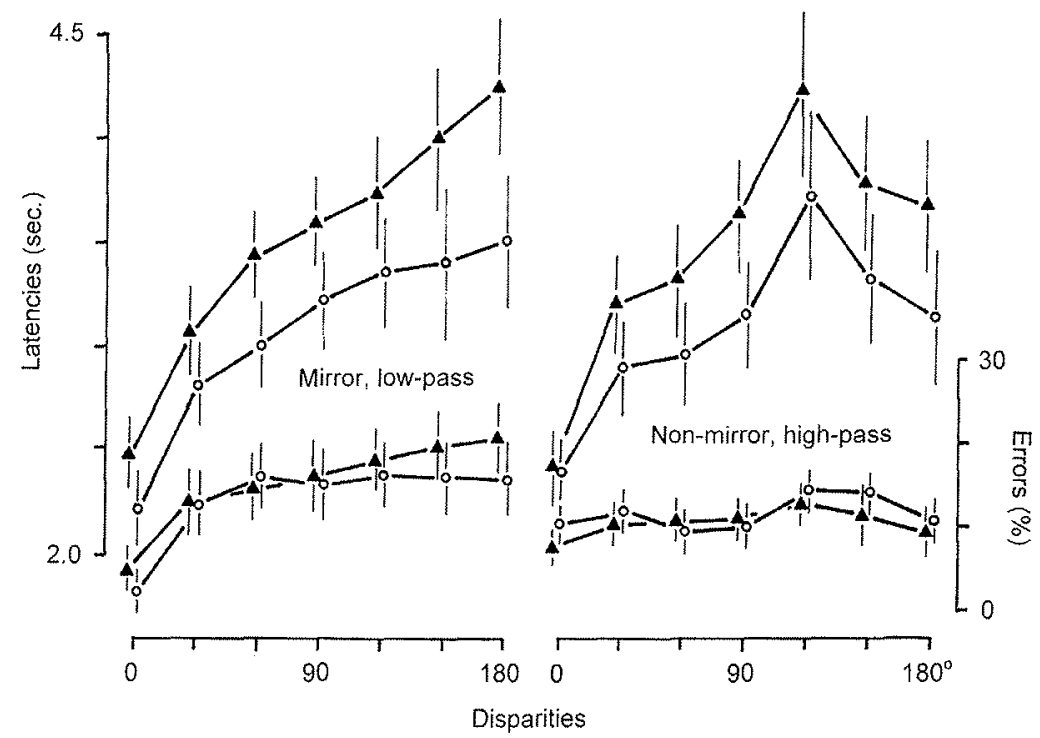

Fig. 4. Mean latencies (sec.) and error rates (\%) \pm standard errors as a function of orientation disparity and subjects' sex [men ( $\mathbf{A})$, women (0)], for mirror-different, low-pass filtered, and non-mirror-different, high-pass filtered conditions

\section{Discussion}

It is patent that the experiment yielded an unusual and significant advantage in mental rotation speed by the women over the men accompanied by an important convex curvilinearity of the response latency-orientation disparity dependencies (cf. Fig. 2). As indicated by the significant interactions of orientation-disparity $\times$ stimulus type and for the filtering factor $\times$ orientation disparity previously reported and the shapes of the latency-disparity functions shown in Fig. 3, it is fairly certain that the various stimulus conditions used in this study yielded a graded variety of latency-disparity functions. It is thus reasonable to attribute the above results to the inclusion of non-mirror-different (Förster, et al., 1996) in addition to mirror-different stimuli and the inclusion of spatially low-pass and high-pass filtered stimuli (new additional conditions) in adjunction to unfiltered stimuli into the design of the present experiment on mental rotation.

This latter attribution is supported by the fact that the curvilinearity of the latency-disparity dependencies was significantly more pronounced in the 
arbitrary, high-pass stimulus condition than in the mirror-different, low-pass condition (cf. Fig. 4). It is pertinent to mention that in contrast, similarly structured experiments which involve only mirror-different and spatially unfiltered stimuli have yielded essentially rectilinear latency-disparity functions and, indeed, faster rotation performance by men than women (Delius \& Hollard, 1995; Raabe \& Delius, unpublished data). Results of the present experiment agree with the assumption that the non-mirror-different stimulus and high-pass stimulus-filtering condition encourages subjects to apply, in addition to the well known holistic rotation strategy, a less discussed analytic rotation strategy (Jolicoeur, et al., 1985). Conversely, the mirror-different stimulus and low-pass stimulus filtering condition appeared to discourage the application of the analytic rotation strategy.

However, the most salient result of this experiment was that the women's mental rotation was significantly faster and performance significantly more curvilinear than that of the men (cf. Figs. 2 and 3). The overall better performance of women than men in rotation speed probably arose because by disposition, as previously argued, females excel in and prefer analytic spatial strategies, while males excel in and prefer holistic spatial strategies (Kylonnen, et al., 1984; Hosenfeld, et al., 1997; see also Karadi, et al., 2003). In the present context the holistic strategy entails that the mental image of the relevant stimulus is rotated at a largely constant angular speed while being continuously checked for whether it matches the percept of the other stimulus. The analytic strategy, on the other hand, entails identification of a feature in one of the relevant stimuli followed by a search for a matching feature in the percept of the other stimulus. Perhaps in this experiment the men predominantly attempted the holistic strategy and only switched to the analytic detection strategy at which they are less efficient when the holistic strategy became inefficient with unsuited stimuli which were presented with large orientation disparities. Conversely, one may suppose that female subjects, less effective with the holistic strategy, genetally preferred the analytic strategy pretty regardless of which type of stimuli were being presented. These women thus mentally rotated generally faster than the men, who presumably were less likely to apply the probably more effective analytic strategy across all conditions.

It is true, however, that females were expected to be relatively better at mental rotation in the non-mirror, high-pass condition and the males to be relatively better at mental rotation in the mirror-different, low-pass condition but that that was not statistically significantly the case. It might be illuminating to conduct an experiment in which the various stimulus conditions would be presented in a blocked manner, a procedure which may be expected to be more effective in separating the contribution of the two strategies than the randomized-conditions used here. With the latter presentation 
participants could not easily adopt the strategy best suited for each different condition because switching of conditions would be ever recurring (cf. Allport, Styles, \& Hsieh, 1994). It could also be valuable to pre-assess participants' strategy preferences with a suitably differential spatial abilities test (Kylonnen, et al., 1984). Such an experiment might better define the source of the frequently reported male superiority in mental rotation performance over that of females. It is worth pointing out, however, that this laboratory has been disappointingly unsuccessful in trying to instruct and train subjects to adopt preferentially either a holistic or an analytic mental rotation strategy (Lüken \& Siemann, unpublished data). Nevertheless, a new avenue of research has been opened for a better understanding of the variants in cognitive strategy that might underlie the sex difference in mental rotation performance.

\section{REFERENCES}

Allport, A., Strues, E. A., \& Hsieh, S. (1994) Shifting intentional set: exploring the dynamic control of tasks. In C. Umiltà \& M. Moscovitch (Eds.), Attention and perfonmance XV. Cambridge, MA: MIT Press. Pp. 421-452.

Collins, D. W., \& Kimura, D. (1997) A large sex difference on a two dimensional mental rotation task. Behavioral Newoscience, $111,845-849$.

Cooper, L. A. (1975) Mental rotation of random two dimensional shapes. Cognitive Psychology, $7,20-43$.

Corbalis, M. C. (1988) Recognition of disotiented shapes. Psychological Review, 95, 115-123.

Dabbs, J. M., Chang, E. L., Strong, R. A., \&Mrun, R. (1998) Spatial ability, navigation strategy and geographic knowledge among men and women. Evolution and Human Bebaviour, 19, 89.98.

Davison, K. K., \& Susman, E. J. (2001) Are hormone levels and cognitive ability related during early adolescence? Intemational Joumal of Behavional Development, 25, 416-428.

Delius, J. D., \& Hollard, V. D. (1995) Orientation invariance in pattetn recognition by pigeons and humans. Joumal of Comparative Psychology, 109, 278-290.

Eme, P. E., \& Marquer, J. J. (1999) Individual strategies in a spatial task and how they relate to aptitudes. European Joumal of Psychology of Education, 14, 89-108.

Farrel, B. (1988) Comparisons requirements and attention in identical-nonidentical stimulus discriminations. Joumal of Experinental Psychology: Human Percepion and Perfommance, $14,707-715$.

FiAhERTY, M. (2005) Gender differences in mental rotation ability in three cultures: Ireland, Ecuador and Japan. Prychologia, 48, 31-38.

Folk, M. D., \& LuCE, R. D. (1987) Effects of stimulus complexity on mental rotation rate of polygons. Joumal of Experimental Psychology: Haman Perception and Perfomance, 13, 395-404.

Förster, B, Gebharot, R. P., Lindlar, K., Siemann, M., \& Delius, J. D. (1996) Mental rotation, a function of elementary stimulus discriminability? Perception, 25, 1301-1316.

Gellerman, L. W. (1933) Chance orders of alternating stimuli in visual discrimination experiments. Joumal of Genetic Psychology, 42, 206-208.

Grimshaw, G. M., Sitarenios, G., \& Finnegan, J. K. (1995) Mental rotation at 7 years: relations with prenatal testosterone levels and spatial experiences. Brain and Cognition, 29 , 85-100.

Hausmann, M., Slabbekoorn, D., Van Goozen, S. H. M., Cohen-Kettenis, P. T., \& GüntürKüN, O. (2000) Sex hormones affect spatial abilities during the menstrual cycle. Bebavioral Netroscience, 114, 1245-1250.

Hochberg, J., \& Gelman, L. (1977) The effect of landmark features on mental rotation times. Memory \& Cognition, 5, 23-26. 
HÖGER, R. (1997) Speed of processing and stimulus complexity in low-frequency and high-frequency channels. Perception, 26, 1039-1045.

Hollard, V. D., \& Delius, J. D. (1982) Rotational invariance in visual pattern recognition by pigeons and humans. Science, 218, 804-806.

Hosenfeld, I. Strauss, B., \& Köll.er, O. (1997) Geschlectsdifferenzen bei Raumvorstellungsaufgaben, eine Frage der Strategie [Gender differences in spatial ability, a question of strategy]? Zeitschrift für Pädagogische Psychologie, 11, 85-94.

Jolicoeur, P, Regehr, S., Smith, L. J. P., \& Smith, G. N. (1985) Mental rotation of representations of two-dimensional and three-dimensional objects. Canadian Journal of Psychology, 39. $100-129$.

Karadi, K., Csatho, A., Kallai, J., \& Labadi, B. (2003) Sex differences of cognitive strategy on mental rotation task. Journal of Cultural and Evolutionary Psychology, 1, 73-79.

KRUEGER, L. E. (1984) Self-termination in same-different judgements. Joumal of Experimental Psychology: Learning, Memory, and Cognition, 10, 271-284.

Kunde, W., \& Hoffmann, J. (2000) Global-local orientation congruency effects in visual search. Quarferly Journal of Experimental Psycbology, A53, 537-548,

Kylonnen, P. C., Lohman, D. F., \& Woltz, D. J. (1984) Componential modelling of alternative strategies for performing spatial tasks. Joumal of Educational Psychology, 76, 1325-1345.

Leone, G., Taine, M. C., \& Droulez, J. (1993) The influence of long-term practice on mental rotation. Cognitive Brain Research, 1, 241-255.

Linn, M. C., \& Peterson, A. C. (1985) Emergence and characterisation of sex differences in spatial ability, a meta-analysis. Child Development, 56, 1479-1498.

Makany, T, Karadi, K., Kallai, J., \& Nadel, L. (2002) Interference between verbal concept formation and spatial mental rotation in fermale subjects. Perceptual and Motor Skills, 95, 227-232.

Mcburney, D. H., Gaulin, S. J. C., Devinen, T., \& Adams, C. (1997) Superior spatial memory of women, stronger evidence for the gathering hypothesis. Evolution and Human Behaviour, 18, 165-174.

NAVON, D. (1977) Forest before trees: the precedence of global features in visual perception. Cogritive Psycbology, 9, 353-383.

Nxborg, H. (1983) Spatial ability in men and women: a review and new theory. Advances in Bebavioural Research and Therapy, 5, 89-140.

PaqueT, L. (1991) Mental rotation of compound stimuli: the effects of task demands, practice and figural goodness. Memory $\&$ Cognition, 19, 558-567.

Pierret, A., \& Perronet, F. (1994) Mental rotation and mirror-image discrimination. Perceptual and Motor Skills, 78, 515.524.

Richter, W., Somorjai, R., Summers, R., Jarmasz, M., Menon, R. S., Gatr, J. S., GeorgopovLOS, A. P., Tegeler, C., Ugurbil, K., \& Kim, S. G. (2000) Motor area activity during mental rotation studied by time-resolved single trial fMRT. Joumal of Cognitive Neuroscience, $12,310-320$.

Robertson, L. C., \& Palmer, S. E. (1983) Holistic processes in the perception and transformation of disoriented figures. Journal of Experimental Psychology: Human Perception and Performance, 9, 203-214.

Schultz, K. (1991) The contribution of solution strategy to spatial performance. Canadian Jounthal of Psychology, 45, 451-462.

Shepard, R. N., \& Cooper, L. A. (1982) Mental images and their transformations. Cambridge, MA: MIT Press.

Shepard, R. N., \& MEtzler, J. (1971) Mental rotation of three-dimensional objects. Science, $171,701.703$.

Shulman, G. L., Sulivan, M. A., Gish, K., \& Sakoda, W. (1986) The role of spatial frequency channels in the perception of local and global structure. Perceptiont, 15, 259-273.

Siemann, M. (1997) Von der Reizdiskrimination zur Schlussfolgerung bei Mensch und Tier [From stimulus discriminations to logical conclusions in bumans and animals]. Habilitation thesis, Univer. of Konstanz.

Takano, Y. (1989) Perception of rotated forms, a theory of information types. Cognitive Psychology, 21, 1-59. 
Tan, Ü., OkJizan, M., Albayrak, T., \& Akgun, A. (2003) Sex differences in verbal and spatial ability reconsidered in relation to body size, lung volume, and sex hormones. Percepiual and Motor Skills, 96, 1347-1360.

TARR, M. J., \& PInkER, S. (1989) Mental rotation and orientation-dependence in shape recognition. Cognition Psychology, 21, 233-282.

Terlecki, M. S., \& Newcombe, N. S. (2005) How important is the digital divide? The relation of computer and videogame usage to gender differences in mental rotation ability. Sex Roles, 53, 433-441.

Tomasino, B., Borroni, P., Isaja, A., \& Rumiati, R. I. (2005) The role of the primary motor cortex in mental rotation: a TMS study. Cognitive Netropsychology, 22, 348*363.

Voxer, D., Voyer, S., \& Bryden, M. P. (1995) Magnitude of sex differences in spatial abilities: a meta-analysis and consideration of critical variables. Psychological Bulletin, 117, 250. 270.

Wohlschläger, A., \& Wohlschläger, A. (1998) Mental and manual rotation. Joumal of Experimental Psychology: Human Perception and Performance, 24, 397-412.

Yuile, J. D., \& Steiger, J. H. (1982) Nonholistic processing in mental rotation. Perception $\mathcal{E}$ Psychophysics, 31, 201-209.

Zimmer, H., \& Fröhlich, W. D. (1991) Merkmalsidentifikation und Mentale Rotation unter Automatisierungsbedingungen [Feature identification and mental rotation under automating conditions]. Zeitschrift fïr angewandte und experimentelle Psychologie, 38, 149-167. 